\title{
Social networking between cells of the foetal skeleton: the importance of thyroid hormones
}

\author{
Colin Farquharson \\ Bone Biology Group, The Roslin Institute, Royal (Dick) School of Veterinary Studies, University of Edinburgh, Edinburgh EH25 9RG, UK \\ (Correspondence should be addressed to C Farquharson; Email: colin.farquharson@roslin.ed.ac.uk)
}

\begin{abstract}
In this issue of Journal of Endocrinology, Lanham et al. investigated the effects of hypothyroidism on the developing skeleton of the ovine foetus in utero. Their analyses indicated that, following thyroidectomy, bone growth, structure and mechanical properties were all altered at late gestation or at term. Adrenalectomy, whilst preventing the
\end{abstract}

prepartum rise in triiodothyronine, did not modify skeletal development. The hypothyroid-mediated skeletal defects of the developing foetus described in this study may have clinical implications for bone health in later life.

Journal of Endocrinology (2011) 210, 135-136
Thyroid dysfunction has unfavourable effects on various organ systems and thus the maintenance of normal thyroid gland status is essential for health in both animals and man. Normal thyroid hormone levels are achieved through the actions of the hypothalamic-pituitary-thyroid axis, a classical endocrine negative feedback loop, resulting in tightly regulated levels of the circulating thyroid hormones thyroxine $\left(\mathrm{T}_{4}\right)$ and triiodothyronine $\left(\mathrm{T}_{3}\right)$. Whilst the prohormone $T_{4}$ is largely inactive, it is the predominant thyroid hormone secreted by the thyroid gland before it is activated by its conversion into $T_{3}$ by the types 1 and 2 iodothyronine deiodinase enzymes (D1 and D2). This conversion produces about $80 \%$ of the active $\mathrm{T}_{3}$ hormone present within the circulation of healthy individuals with the balance coming directly from the thyroid gland itself. Furthermore, essential fine-tuning of $\mathrm{T}_{3}$ levels occurs within the cell through the relative activities of D2 and D3 which determines the local intracellular levels of $\mathrm{T}_{3}$ before its binding to nuclear receptors. Both D2 and D3 are important modulators of thyroid hormone responsiveness and their relative cellular activities provide a mechanism by which target cells can control the timing of cellular responses to $T_{3}$ in a cell/tissue-specific manner.

Hypothyroidism, first identified by Thomas Curling in 1850 , is most commonly caused by an autoimmune condition in which the thyroid gland is slowly destroyed (primary hypothyroidism or Hashimoto's thyroiditis). Although there is a resulting panoply of clinical manifestations it has long been recognised from animal models and population studies that the skeletal system is exquisitely sensitive to systemic thyroid status. New data has also indicated that osteoblast D2 may act to buffer the effects of lowered circulating thyroid levels on the skeleton (Bassett et al. 2010). In the adult skeleton, hypothyroidism results in altered bone maintenance and fracture risk through effects on osteoblast- and osteoclastmediated bone turnover. On the contrary, in children and young postnatal animals hypothyroidism delays skeletal development causing growth arrest, short stature and impaired bone maturation (Rivkees et al. 1988, Bassett \& Williams 2008). These disadvantageous effects on stature are through the well-recognised actions of $\mathrm{T}_{3}$ on growth plate chondrocyte proliferation and differentiation leading to impaired endochondral ossification.

Less, however, is known about the precise nature of the skeletal abnormalities that occur as a consequence of hypothyroidism during embryonic development. Congenital hypothyroidism affects $\sim 1$ in 4000 births in the UK and is commonly caused by defective thyroid gland development. Despite recognising that congenitally hypothyroid infants have delayed bone maturation and shorter long bones the precise role of $\mathrm{T}_{3}$ in the control of bone development and structure in the foetus is unclear (Sack et al. 1993). Hypothyroidism in utero may manifest itself differently in the skeleton from that noted in juveniles and adults due to the low levels of plasma $T_{3}$ and the high activity of D3 in the placenta, endometrium and many foetal tissues. This low exposure to active $\mathrm{T}_{3}$ is required for normal development and growth of the foetus. This situation is known to change near 
term when there is an increase in circulating levels of $\mathrm{T}_{3}$ and increased D2 activity results in a greater conversion of $T_{4}$ to $\mathrm{T}_{3}$ within target cells.

A detailed description of the effects of hypothyroidism on the foetal skeleton has now been addressed in a study published in this issue of Journal of Endocrinology (Lanham et al. 2011). The choice of the sheep model in this study of congenital hypothyroidism is very shrewd as it is recognises that the temporal development of the thyroid axis is similar in both sheep and man. This animal model thus helps us understand the skeletal mechanisms that are disrupted when normal development goes wrong as a consequence of hypothyroidism and thereby provides basic knowledge relevant to human health.

Thyroidectomy at 105-110 days of gestation resulted in very low levels of foetal plasma $\mathrm{T}_{4}$ and $\mathrm{T}_{3}$ (no prepartum rise in foetal plasma $T_{3}$ ) and the foetuses had shorter bones and altered metatarsal structure and strength when analysed at or close to term (145 \pm 2 days). The effects of thyroidectomy on long bone growth are similar to that observed in children and juvenile animals and are consistent with the known effects of $\mathrm{T}_{3}$ deficiency on growth plate dynamics. The authors tested the strength of both cortical and trabeculae compartments and found that trabecular bone from thyroidectomised sheep was stronger although more stiff and brittle. No effects of thyroidectomy on cortical bone thickness or strength were noted. The width of individual trabeculae was also greater in the hypothyroid sheep that has also been reported in adult hypothyroidism in man. The thyroidectomised sheep data, at and just before term, indicated that the structural changes in bone were a result of impaired bone formation, whilst resorption was normal. The reduced formation may be a consequence of altered signalling pathways such as the GH/IGF1 axis that is known to be anabolic to the skeleton. These changes resemble the phenotype reported in adult D2 knockout mice but the precise cellular basis of $\mathrm{T}_{3}$ on specific bone cells requires further study.

Interestingly, adrenalectomy that, as expected, blunted the prepartum rise in plasma $T_{3}$ without altering $T_{4}$ levels had no effect on bone structure or strength and therefore raises questions about the physiological importance of the prepartum rise in plasma $T_{3}$ on skeletal development during late gestation. Locally regulated D2 may be able to maintain $\mathrm{T}_{3}$ concentrations in osteoblasts of adrenalectomised foetuses but it may be beyond the capacity of this local feedback mechanism to compensate in cases of such overt hypothyroidism.

The data reported in this paper is important as it provides significant insight into the effects of hypothyroidism on the various skeletal compartments in an animal model that has relevance to man. The effects of congenital hypothyroidism on the long-term health of the juvenile and adult skeleton, even if treated, is unclear as foetal skeletal programming and development are known to have significant bearing on bone quality in later life. This, therefore, remains an area for further fruitful research.

\section{Declaration of interest}

The authors declare that there is no conflict of interest that could be perceived as prejudicing the impartiality of the research reported.

\section{Funding}

This research did not receive any specific grant from any funding agency in the public, commercial or not-for-profit sector.

\section{Acknowledgements}

The author acknowledges Institute Strategic Programme Grant Funding from the BBSRC for support.

\section{References}

Bassett JHD \& Williams GR 2008 Critical role of the hypothalamicpituitary-thyroid axis in bone. Bone 43 418-426. (doi:10.1016/j.bone. 2008.05.007)

Bassett JHD, Boyde A, Howell PGT, Bassett RH, Galliford TM, Archanco M, Evans H, Lawson MA, Croucher P, St Germain DL et al. 2010 Optimal bone strength and mineralization requires the type 2 iodothyronine deiodinase in osteoblasts. PNAS 107 7604-7609. (doi:10.1073/pnas. 0911346107)

Lanham SA, Fowden AL, Roberts C, Cooper C, Oreffo ROC \& Forhead AJ 2011 Effects of hypothyroidism on the structure and mechanical properties of bone in the ovine fetus. Journal of Endocrinology 210 63-71. (doi:10.1530/ joe-11-0138)

Rivkees SA, Bode HH \& Crawford JD 1988 Long-term growth in juvenile aquired hypothyroidism - the failure to achieve normal adult stature. New England Journal of Medicine 318 599-602. (doi:10.1056/NEJM198 803103181003 )

Sack J, Kaiserman I \& Siebner R 1993 Maternal-fetal T(4) transfer does not suffice to prevent the effects of in-utero hypothyroidism. Hormone Research 39 1-7. (doi:10.1159/000182686)

Received in final form 14 June 2011

Accepted 16 June 2011

Made available online as an Accepted

Preprint 16 June 2011 